\title{
New Scopes for Practice - Interdisciplinary Webinars for Emergency Medicine and Biomedical Informatics - Health Informatics
}

\author{
Kaija SARANTO ${ }^{\mathrm{a}, \mathrm{b}, 1}$, Catherine CHRONAKI ${ }^{\mathrm{a}, \mathrm{c}}$, Luis GARCIA-CASTRILLO \\ RIESGO $^{\mathrm{d}}$, Louise B. PAPE-HAUGAARD ${ }^{\mathrm{a}, \mathrm{e}}$ and John MANTAS ${ }^{\mathrm{a}, \mathrm{f}}$ \\ ${ }^{a}$ European Federation of Medical Informatics, Switzerland \\ ${ }^{b}$ Department of Health and Social Management University of Eastern Finland, Finland \\ ${ }^{c}$ HL7 Europe, Brussels, Belgium \\ ${ }^{d}$ European Society for Emergency Medicine, Belgium \\ ${ }^{e}$ Department of Health Science and Technology, Aalborg University, Denmark \\ ${ }^{f}$ National and Kapodistrian University of Athens, Greece
}

\begin{abstract}
This paper presents the early outcomes of the educational cooperation between two European academic associations, namely the European Federation of Medical Informatics (EFMI) and European Society of Emergency Medicine (EUSEM). Two webinars were organized in December 2019 and June 2020 to explore areas where mutual education would be beneficial for interdisciplinary cooperation to advance the digitization of emergency departments for the benefit of patients, health professionals and the health system as a whole. Preliminary findings from the analysis of these two webinars are presented and the steps for further cooperation are outlined.
\end{abstract}

Keywords. education, webinars, evaluation, interdisciplinary cooperation, competencies

\section{Introduction}

EUSEM (European Society for Emergency Medicine) and EFMI (European Federation Medical Informatics), signed an agreement to collaborate on research and education to advance the digital transformation of emergency departments throughout Europe. [1-2] As a part of the agreement members of EFMI Working Group of Education and EUSEM Research Committee decided to design educational offerings in the form of virtual seminars (i.e. webinars) on topics advancing the digital transformation of emergency departments through the paradigm of biomedical and health informatics science and technology.

Interdisciplinary collaboration is a concept used to convey when two or more scientific fields integrate methods, knowledge and skills, theories, perspectives, and

1 Corresponding author, Dr. Kaija Saranto, P.O. Box 1627, 70211 Kuopio, Finland, kaija.saranto@uef.fi 
different disciplinary knowledge bodies, to realise innovative solutions and new insights in areas where new scopes or practices are needed. [3-5]

Professional competencies are regulated based on national legislation and European level agreements. Literature also highlights the importance of continuous personal development in an expert area to release pressures in professional competencies. [6] Implementation of electronic health records is a key area of education needs. However, after implementation a lot of updates and extra tools need adoption to mainstream their use in daily practice. [5]

Digitization of continuing education offers new ways of organizing education sessions. This trend has been further accelerated by the recent COVID-19 pandemic. Health care professionals working in hectic environments may have difficulties to participate in-service training that often is restricted to most important areas related to work practices. [7] Virtual seminars or Webinars are an effective way to organize education for a wide audience and maintain recordings for offline viewing. EFMI and EUSEM cooperated to advance the digital transformation of Emergency Departments, an area where the need for education, skills development and capacity building is recognised.

The following research questions are addressed in this paper based on our experiences from the two interdisciplinary webinars co-organized by EFMI and EUSEM: (1) How do participants from two scientific fields assess their experiences from the joint webinars? (2) What are the areas for development for future webinars? (3) What are the opportunities for long term cooperation of EUSEM and EFMI?

\section{Methodology}

In this section, the process of cooperation in organising the EUSEM-EFMI webinars is described with aims, structure, and content including an assessment plan. The committee defined aims of the webinars and more detailed objectives for the two webinars in the joint meeting at the EUSEM conference in autumn 2019. Under the leadership of President Luis Garcia-Castrillo Riesgo from EUSEM and Vice President Catherine Chronaki from EFMI two webinars were agreed to be organized jointly under the auspices of the Education WG of EFMI and the Research committee of EUSEM. The EUSEM Academy and the EFMI website were to host the recorded webinars for those unable to participate when the webinars are originally broadcasted.

The aims of both webinars were set based on the title, "Structured data, Big data, Health analytics, and Clinical decision support". A guiding question to focus on the content and stimulate the interest of the audience was added: "How does it change emergency medicine?"

The specific aims of the first webinar were to help the participants:

- to identify the special requirements of emergency care in terms of providing urgent medical care to patients

- to identify the tools and knowledge needed for decision making in the demanding environment of the Emergency Departments (ED)

- to familiarize with current evidence on structuring electronic health records and understand the purpose and role of clinicians in structured documentation development 
- to demonstrate that it is possible to implement and successfully adopt centralized integrated and shared electronic prescription services for health care on national level based on the Finish experience.

The aims for the second webinar were to help the participants:

- to define the key concepts: Big Data, Artificial Intelligence, and eHealth in terms of achievements, opportunities, and benefits of their use in healthcare

- to present how current use of data can be prepared and used for data analytics and $\mathrm{AI}$ in emergency care/emergency medicine

- to understand the sources and management of diagnostic error in the ED

- to evaluate the potential of computerized diagnostic decision support (CDDS) in the ED, while understanding the limitations of current CDDS.

The speakers for the webinars were selected among the experts from both organisations. Each of them represented both national and clinical expertise in the field of health and medical informatics or emergency medicine. The length of the webinars were 60 minutes each and their structure was as follows: introduction, presentations, Questions and Answers (Q\&A), and discussions. Polls were used to activate the participants and a moderator was monitoring the Q\&A option. Chat and microphone were disabled for the viewers during the presentation.

An assessment tool was designed to collect feedback from the participants. The tool had questions about the background of participants, about the usefulness and satisfaction of the webinar content (Likert scale from 1 to 5), and the intent of participants to participate in future webinars on specific topics of joint interest. The second webinar also gathered information about the pandemic situation in terms of their intention to join webinars or other online educational events more often. The assessment tool was available after the webinars.

\section{Results}

\subsection{Participant experiences}

The number of unique on-line viewers was 45 in the first and 74 in the second webinar. The participants represented both scientific fields equally in the first webinar. They were experienced viewers in $40 \%$ (first webinar $=\mathrm{FW}$ ) and $26 \%$ (second webinar $=$ SW) having attended webinars more than 6 times already. For more than $30 \%$ (FW) and $46 \%$ (SW) of viewers this was their first webinar. In terms of their intention to join webinars or other online educational events more often due to pandemic situation all viewers agreed. Professional interest was the main reason to participate in the webinars. The next most reported reason 'because informatics is a hot topic within my institute'. Viewers also argued their participation 'because I feel I need to develop myself on this topic or out of personal interest'.

The attendees represented Clinical Emergency Medicine and Medical Informatics equally (44\%) in the FW. Most of the attendees (73\%) in the SW came from Clinical Emergency Medicine and 13 percent of viewers were from Biomedical and Health Informatics (BMHI). They regarded that each speaker gave high quality information, the presentations were useful (mean 4) and the level of information was adequate (mean 4). The viewers were overall satisfied of the webinar (mean 4). The number of speakers was assessed as proper. 


\subsection{Future development}

The viewers expressed their interest to participate in the next webinars: $80 \%$ (first webinar $=\mathrm{FW}$ ) and $90 \%$ (second webinar $=\mathrm{SW}$ ). Regarding future topics the viewers would like to have are presented in the figure 1.

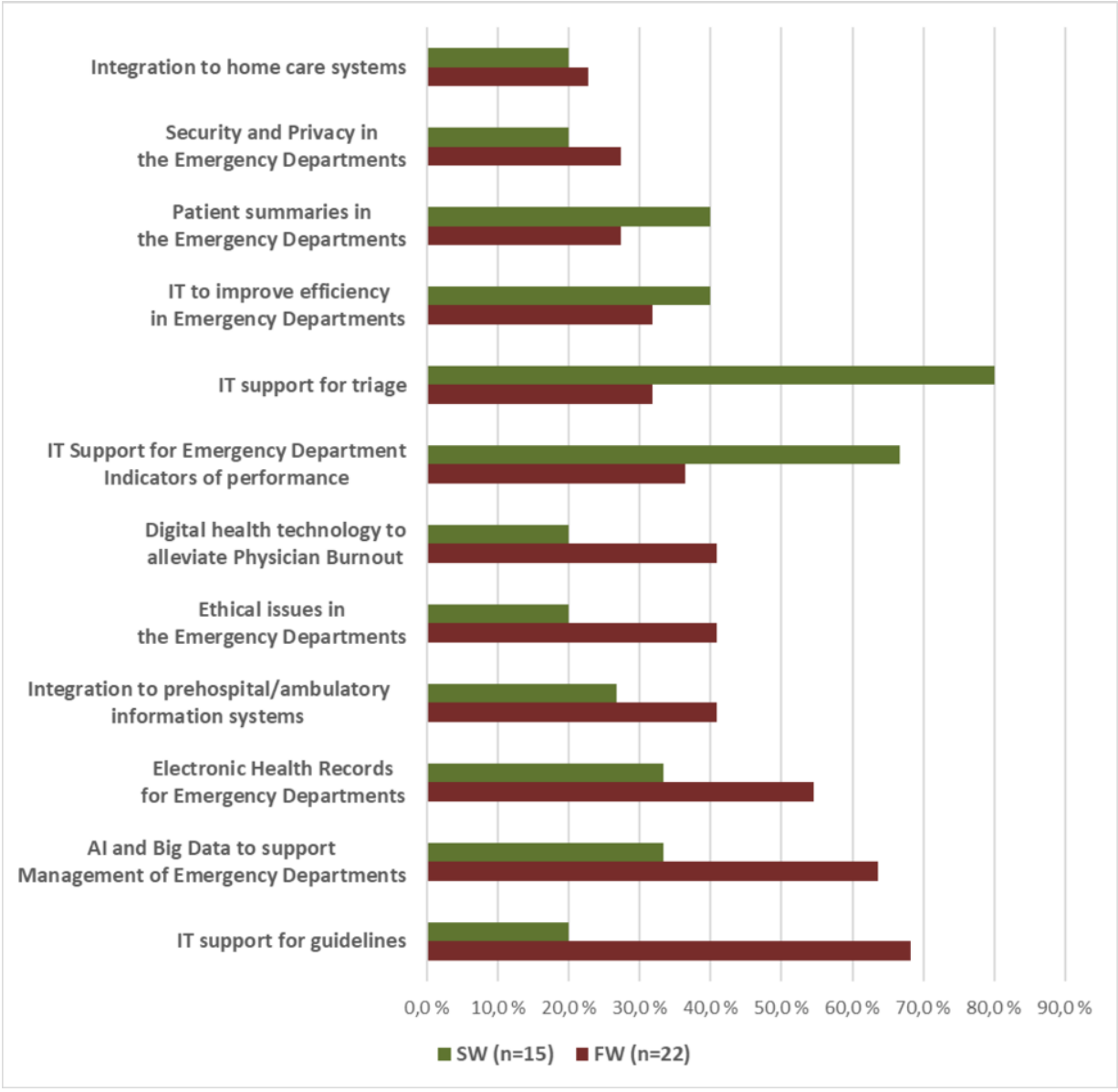

Figure 1. The topics of interest among the webinar viewers

\section{Discussion and Conclusion}

Interdisciplinary education is challenging not only due to difficulty to choose content of high relevance for specific audience, but also due to new insights in areas where new scopes or practices are emerging [4]. BMHI is not an unknown discipline for professionals in Emergency Medicine or vice versa. However, each discipline has its own concepts terms, and theories, which need to be introduced to establish baseline understanding and be able to elicit rewarding outcomes in continuing education $[3,5]$. Based on our experience, the interdisciplinary cooperation worked extremely well. The speakers were invited based on carefully selected topics and they all received excellent 
feedback from the viewers. Online seminars or webinars are demanding to plan in terms of content, structure, and cooperation with the viewers, partly because it is difficult to maintain the attention of viewers for a long time [7]. We used polls to activate them and stimulate their interest. The speakers answered one or two questions, a number typical for conference venues.

The viewers represented equally both scientific fields in the first webinars but in the second most of the viewers were from emergency medicine. They were also less experienced viewers than those presented in the first webinar. The topics suggested for future webinars indicate the appetite of emergency medicine professionals to gain knowledge about recent trends in the digitization of emergency medicine and its impact in their work.

The number of registrations for the webinars was almost double for both webinars compared to those who actually participated. However, the number of viewers on the on-line webinars doubled in the second webinar. This is probably the case because the professionals may have other commitments late afternoon which was the on-line time for the webinars.

The webinars are available on the EUSEM Academy site. For the time being (July 2020) the first webinar was viewed 99 times, and the second one 53 times on the EUSEM Academy site. The webinars were the first actions in the joint agreement between EFMI and EUSEM. The success of these first webinars are encouraging and EUSEM and EFMI plan to continue their cooperation for the years to come.

\section{Acknowledgements}

The authors want to thank the distinguished speakers of the two webinars and Mrs. Willemijn van Hees, Project Manager at EUSEM for her extensive support in preparing and launching the webinars.

\section{References}

[1] European Federation of Medical Informatics (EFMI). https://efmi.org/ Accessed July $2^{\text {nd }}, 2020$

[2] European Society of Emergency Medicine (EUSEM). https://eusem.org/ Accessed July 2 $2^{\text {nd }}, 2020$

[3] Menken S, Keestra M. An introduction to interdisciplinary research: theory and practice. Amsterdam: Amsterdam University Press, 2016.

[4] Klaassen RG. Interdisciplinary education: a case study. European Journal of Engineering Education. 2018; 43(6): 842-859. https://doi.org/10.1080/03043797.2018.1442417

[5] Patel V, Yoskowitz NA, Arocha JE, Shortliffe EH. Cognitive and learning sciences in biomedical and health instructional design: A review with lessons for biomedical informatics education. Journal of Biomedical Informatics. 2009; 42(1): 176-197.

[6] Mantas J, Ammenwerth E, Demiris G, Hasman A, Haux R, Hersh W, Hovenga E, Lun KC, Marin H, Martin-Sanchez F, Wright G. IMIA Recommendations on Education Task Force. Recommendations of the International Medical Informatics Association (IMIA) on Education in Biomedical and Health Informatics. First Rev. Methods Inf Med. 2010; 49(2): 105-120.

[7] Nadama1 HH, Tennyson M, Khajuria A. Evaluating the usefulness and utility of a webinar as a platform to educate students on a UK clinical academic programme. J R Coll Physicians Ed 2019; 49: $317-22$. 